\title{
Aspirin ASPIREs to reduce vascular events after VTE
}

Low-dose aspirin reduces the risk of major vascular events after unprovoked venous thromboembolism (VTE), according to data from the ASPIRE trial, which were presented in November 2012 at the AHA Scientific Sessions. Long-term therapy with a vitamin $\mathrm{K}$ antagonist such as warfarin reduces VTE recurrence, but this drug is inconvenient and associated with an increased risk of bleeding. Aspirin, therefore, might be a

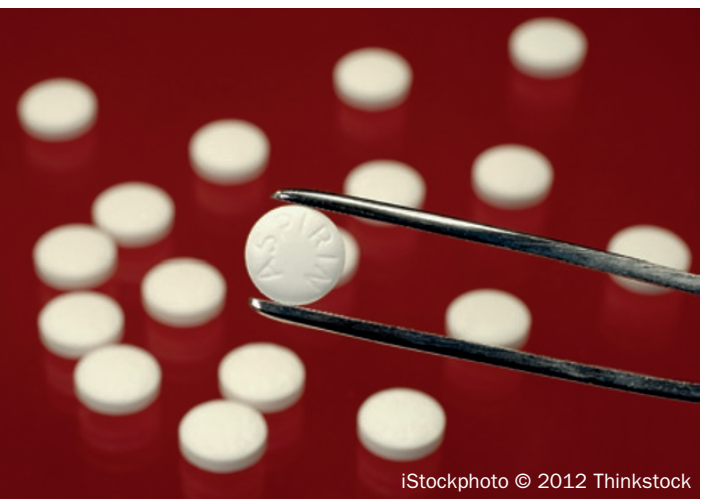

simple and inexpensive alternative for thromboprophylaxis in these patients.

Investigators in the ASPIRE trial recruited 822 patients who had experienced a first, unprovoked VTE (not attributable to confinement in bed, major surgery or trauma, pregnancy, or the use of hormonebased medications). They had all completed initial anticoagulant therapy (heparin followed by warfarin for a minimum of 6 weeks), and were then randomly allocated to receive $100 \mathrm{mg}$ of aspirin daily or a placebo for up to 4 years (median duration of follow-up 37.2 months).

The rate of the primary end point, recurrent VTE, was not significantly different between the groups ( $4.8 \%$ vs $6.5 \%$ per year; HR 0.74, 95\% CI 0.52-1.05). However, aspirin reduced the rate of two composite secondary end pointsVTE, myocardial infarction, stroke, or cardiovascular death $(5.2 \%$ vs $8.0 \%$ per year; HR 0.66, 95\% CI 0.48-0.92, $P=0.01$ ), and VTE, myocardial infarction, stroke, major bleeding, or all-cause death $(6.0 \%$ vs
9.0\%; HR 0.67, 95\% CI 0.49-0.91, $P=0.01$ ). The rate of bleeding and of serious adverse events did not differ between the groups.

Researchers originally planned to enroll 3,000 patients, but this target was reduced because of slow recruitment, and the study might have been underpowered to detect a reduction in the primary outcome. When, as prospectively planned, the data were combined with those from the similar WARFASA study, the rate of recurrent VTE and of major vascular events was significantly reduced by $32 \%$ and $34 \%$, respectively. Dr Timothy Brighton, lead author on the trial report, says that the investigators now plan "to develop a way to predict in advance which patients with unprovoked thrombosis will benefit from anticoagulation or low-dose aspirin".

Gregory B. Lim

Original article Brighton, T. A. et al. Low-dose aspirin for preventing recurrent venous thromboembolism. N. Engl. J. Med. doi:10.1056/NEJMoa1210384 